\title{
Aseptic meningitis after posterior fossa surgery treated by pseudomeningocele closure
}

\author{
C E M Hillier, A Penrose Stevens, F Thomas, J Vafidis, R Hatfield
}

\begin{abstract}
Aseptic meningitis is a recognised complication after posterior fossa surgery. It is often self limiting but occasionally runs a protracted course requiring repeated CSF examination to exclude infection, and treatment with systemic steroids. A patient is described with aseptic meningitis after posterior fossa surgery who underwent posterior fossa re-exploration nearly 3 years after the initial operation. This disclosed a pseudomeningocele, which was closed. The patient remains symptom free almost 2 years after closure. In this case of chronic aseptic meningitis after posterior fossa surgery, closure of the pseudomeningocele found at exploratory surgery led to resolution of the symptoms. (f Neurol Neurosurg Psychiatry 2000;68:218-219)
\end{abstract}

Keywords: aseptic meningitis; pseudomeningocele; posterior fossa

Aseptic meningitis is a syndrome defined by pyrexia and signs of meningism associated with CSF pleocytosis, increased CSF protein, and sterile blood and CSF culture It is a recognised complication of posterior fossa surgery and is usually short lived. We describe for the first time a case associated with a postoperative pseudomeningocele which when closed led to the patient becoming symptom free.

Department of Neurology, University Hospital of Wales, Heath Park, Cardiff CF4 4XW, UK C E M Hillier

$\mathrm{F}$ Thomas

Department of Neurosurgery A P Stephens

J Vafidis

R Hatfield

Correspondence to: Dr C E M Hillier,

Department of Neurology, University Hospital of Wales, Heath Park, Cardiff CF4 4XW, UK

email

charlie.hillier@cdsc.wales.nhs.uk

Received 19 April 1999 and in revised form

21September 1999

Accepted 1 October 1999 phocytes), protein of $0.82 \mathrm{~g} / \mathrm{l}$, CSF glucose of $2.3 \mathrm{mmol} / 1$, and blood glucose of $5.1 \mathrm{mmol} / 1$. Gram stain, bacterial antigen tests for Haemophilius influenzae type b, Streptococcus pneumoniae $N$, meningitidis groups ACYW 135, group b, Streptococcus group B, complement fixation tests, and tissue cultures for viruses, bacterial culture, and acid fast bacilli were negative. A right parietal ventriculoperitoneal shunt was inserted after persistently raised CSF pressures were demonstrated on repeated lumbar puncture and intravenous cefuroxime was started. Despite this, the CSF white cell count remained raised, and the shunt was removed and replaced by an external ventricular drain. Coagulase negative staphylococci were isolated from the CSF. A second shunt was inserted after the infection had been treated with a 3 week course of intrathecal gentamicin, vancomycin, and intravenous teicoplanin. A repeat cranial CT in February 1995 showed a pseudomeningocele at the site of the scar, and that the shunt was misplaced. The shunt was revised. His CSF at the time of operation showed a white cell count of 920 cells $/ \mathrm{cmm}$ (45\% polymorphs, $55 \%$ lymphocytes), protein $1.72 \mathrm{~g} / 1$, a CSF glucose concentration of $25 \mathrm{mmol} / 1$, and blood glucose of $6 \mathrm{mmol} / 1$.

Over the next year the patient remained unwell and in January 1996 the shunt was removed; however, despite this he continued to complain of an intermittent headache and low back pain and required admission to hospital on 10 separate occasions. Each episode was associated with increase in the size of the pseudomeningocele confirmed both clinically and by cranial CT on several admissions. On each admission he underwent lumbar puncture: measured CSF pressures were intermittently raised $(3-30 \mathrm{~cm})$, the CSF appeared turbid, and microscopy showed a raised white cell count, raised protein, and reduced CSF glucose. The CSF remained aseptic on every admission, microscopy for acid fast bacilli and Cryptococcus, and CSF culture for acid fast bacilli were negative. Complement fixation tests for Herpes simplex and Varicella zoster, and serological testing for fungal precipitins and a labelled white cell scan were also negative. After each lumbar puncture his clinical symptoms improved but only for about 5 days. To exclude a focus of infection, his back pain was investigated with whole spine MRI, which 
demonstrated an enhancing nodule in the distal lumbosacral theca. At an exploratory operation (April 1997), the nerve roots were found encased in fibrotic tissue. The histopathology was in keeping with arachnoiditis: the patient was started on dexamethasone (2 $\mathrm{mg}$ four times daily) for 2 weeks with improvement of his headache. Reduction of the dose resulted in recurrence of his symptoms.

In July 1997, nearly 3 years after posterior fossa craniotomy, the patient underwent repair of the pseudomeningocele as a last resort. After this procedure, he became asymptomatic, required no medication, and has not required further admissions

\section{Discussion}

The relation between cranial surgery and aseptic meningitis has been known for a long time. As early as 1928, Cushing and Bailey ${ }^{1}$ commented that waves of pyrexia suggesting meningitis were "not uncommon following cerebellar operations". Although aseptic meningitis has been noted after supratentorial surgery, it is more frequent after posterior fossa surgery ${ }^{2}$ and seems to occur more commonly in children. It occurs with a greater frequency in patients operated on for non- tumorous abnormalities compared with those operated on for tumour.

Carmel et $a l^{\beta}$ described aseptic meningitis in $70 \%$ of 50 children undergoing posterior fossa surgery. The meningitic reaction did not correlate with the underlying lesion, closure of the dura, initial postoperative CSF red cell count, or postoperative drainage of CSF. Symptoms appeared as late as the 2nd or 3rd week postoperatively and could last 3-4 months. Clinical characteristics described included headaches, fever, and meningism, and less commonly impairment of consciousness and neurological deficit. Pleocytosis in the CSF, with a significant mononuclear component (or less commonly polymorphs but later lymphocytes), often predominated

The underlying aetiology remains uncertain. Cushing and Bailey ${ }^{1}$ suggested that the pyrexia might be due to blood in the CSF but Carmel et $a l^{\beta}$ demonstrated that red blood cells need not be present for pyrexia to continue. Finlayson and Penfield ${ }^{4}$ suggested that a cyst-like cavity may form postoperatively and fill with clot and products of tissue destruction which would discharge periodically, and Matson ${ }^{5}$ proposed that breakdown products from the tumour bed might be responsible. Attempts to define CSF substances associated with aseptic meningitis have included blood/brain/tumour and muscle markers (haemoglobin, bilirubin, myoglobin, and creatinine kinase) but no clear pattern has emerged: operative technique, postoperative radiotherapy, or antibiotics did not influence the course of aseptic meningitis. ${ }^{6}$

Treatment of aseptic meningitis by decompression of a related pseudomeningocele has been previously reported by Jacobs et al. ${ }^{7}$ They describe a case of aseptic meningitis in an 8 year old boy after removal of a cystic astrocytoma. One month after initial surgery he recquired the placement of a ventriculoauricular shunt which became necessary to eliminate the "unsightly bulging of the decompression site." The symptoms resolved after the operation.

To our knowledge, we describe the first case of aseptic meningitis treated by formal closure of a related pseudomeningocele. The finding that pseudomeningocele formation is more common in surgery of the posterior fossa may explain the increased incidence of aseptic meningitis in this group of patients. In the case described, closure of the pseudomeningocele led to resolution of symptoms. Why this occurred is not immediately obvious but hypotheses include the possibility that alteration of normal CSF flow in and around the meningocele leads to the accumulation of, as yet, undetectable inflammatory mediators that are responsible for the meningitic reaction seen. It may be of interest to sample CSF from the meningocele and lumbar region simultaneously and compare concentrations of inflammatory mediators.

1 Cushing H, Bailey P. Tumours arising from blood vessels of the brain: angiomatous malformation and haemangioblastomas. brain: angiomatous malformati

2 Ross D, Rosegay H, Pons V. Differentiation of aseptic and bacterial meningitis in postoperative neurosurgical patients. F Neurosurgery 1988;69:669-74

3 Carmel PW, Fraser RAR, Stein BM. Aseptic meningitis folowing posterior fossa surgery in children. $\mathcal{F}$ Neurosurgery 1974;41:44-8.

4 Finlayson AI, Penfield W. Acute post-operative aseptic leptomeningitis: review of cases and discussion of pathogenesis. Archives of Neurology and Psychiatry 1941;46:250-76.

5 Matson DD. Neurosurgery of infancy and childhood. Springfield: Thomas, 1969 .

6 Kaufman HH, Carmel PW. Aseptic meningitis and hydrocephalus after posterior fossa surgery. Acta Neurochir (Wien) 1978;44:179-96

7 Jacobs GB, Klemme WM, Murphree HC. Aseptic meningitis and pseudomeningocele formation as a complication of surgery in the posterior fossa. Pacific Medicine and Surgery 1966;6:320-4. 